\title{
Pulsating flow and heat transfer in wavy channel with zero degree phase shift ${ }^{\S}$
}

\section{Harun Zontul ${ }^{*}$, Nazım Kurtulmuş², Beşir Şahin ${ }^{3}$}

${ }^{1,3}$ Çukurova University, Mechanical Engineering Department, Turkey

${ }^{2}$ Adana Science and Technology University, Turkey

\begin{abstract}
In this study, heat transfer enhancement of laminar pulsating flow in wavy channel is investigated numerically. The wavy channel has constant wall temperature and its geometric parameters are fixed. Finite volume method based on SIMPLE technique is used to solve governing equations. Simulations performed for Reynolds numbers in the range of $200 \leq \mathrm{Re} \leq 800$. The effects of pulsation frequency is investigated for Strouhal numbers, (St) of $0.05,0.15$ and 0.25 . The differences between flow structures of pulsating and steady flow are discussed. Results indicate that the pulsating flow significantly enhances heat transfer.
\end{abstract}

Keywords: Convection heat transfer, internal fluid flow, fluid mixing, pulsating flow, wavy channels,

\section{INTRODUCTION}

Pulsating flow has attracted the attention of many researchers from view point of its effect on heat transfer. There are many numerical and experimental studies that investigate the heat transfer performance of pulsating flow through the different geometries. Since internal forced convection phenomena has great importance in numerous engineering applications, studies about pulsating flow and heat transfer in channels, ducts and tubes came into prominence.

Researches about heat transfer in flat channels and pipes under pulsating flow condition points out that the effect of pulsating flow is limited in these geometries [1-5]. Depending on flow parameters, heat transfer can be enhanced or reduced. Guo and Sung [1] numerically investigated heat transfer in pipe for the Reynolds number value of 500. Their results showed that for small pulsation amplitude values both heat transfer augmentation and reduction was observed depending on a pulsation frequency. But when pulsation amplitude was large, heat transfer was always enhanced. Chattopadhyay et al. [2] conducted numerical investigation for laminar pulsating flow in a tube. They concluded that pulsating flow has no considerable effect on heat transfer for investigated amplitude and frequency values. Another numerical analysis performed by Rahgoshay et al. [3] for investigation of the effect of flow pulsation on heat transfer in the isothermally heated pipe. In this study nanofluid was used as working fluid. They obtained only small amount of increase in heat transfer within the range of chosen parameters. Pulsating turbulent flow in pipe was numerically investigated by Wanh and Zhang [4]. They observed significant heat transfer enhancement with large pulsation amplitude values. They also showed that there is an optimum Womersley number (Wo) value that maximizes the heat transfer enhancement. Mehta and Khandekar [5] experimentally investigated pulsating laminar flow in square mini-channel.

In their study pulsation amplitude $\left(\mathrm{A}_{\mathrm{p}}\right)$ and the Reynolds number kept constant and change of heat transfer rate with pulsation frequency was observed. It was found that the low frequency values deteriorates heat transfer and higher frequencies cause a slight improvement in heat transfer.

Unlike its limited performance at flat channels and tubes, this type of flow provides notable heat transfer enhancement for cooling of blunt bodies in channels [6-8]. Moon et al. [6] carried out experimental study to show how flow pulsation effects heat transfer from heated blocks in a channel. Imposing flow pulsation causes considerable increase in heat transfer from blocks and it is observed that rate of heat transfer is dependent on the pulsation frequency and inter block spacing. Ji et al. [7] discussed heat transfer from a square cylinder in pulsating flow. This study revealed the emergence of lock-on regime and its effect on heat transfer for pulsating flow. Results showed that when pulsation frequency is doubled the natural frequency, heat transfer enhancement becomes maximum. Kim et al. [8] numerically simulated cooling of electrical components in channel by using pulsating flow. They stated that there is an optimal frequency value and it depends on the cooled geometry.

\footnotetext{
*Corresponding authour

Email: hzontul@cu.edu.tr (H. Zontul)

$\S$ This paper was presented at the IMSEC-2016
} 
Another efficient use of pulsating flow is seen at heat transfer from corrugated channels. Studies about corrugated channels are important in terms of investigation the efficiency of pulsating flow in heat exchangers. In the experimental study of Jin et al. [9] characteristic of pulsating flow in triangular channel was observed. They showed the mechanisms that cause heat transfer enhancement in this channel. Nandi and Chattopadhyay [10] performed a numerical study for wavy micro channel. They reported that even at the low Reynolds number value, pulsating flow causes noticeable heat transfer augmentation in this channel. Akdağ et al.[11] numerically investigated heat transfer enhancement capability of laminar pulsating flow with use of nanofluid. Their study indicated that high volume fraction of nanofluid and a low frequency of pulsation enhances heat transfer performance. An extensive numerical study about pulsating flow in wavy channel presented by Jafari et al. [12]. They investigated effect of two different nanofluids, pulsation frequency, amplitude and the Reynolds number. They reported that pulsating flow is more effective for pure fluid than nanofluid and flow oscillation gives better performance at higher Reynolds numbers. They also observed that there is a linear relationship between amplitude of pulsation and heat transfer enhancement. Alawadhi and Bourisli [13] studied periodic vortex shedding caused by pulsating flow inside wavy channel. They stated that when the frequency of pulsation is close to vortex shedding frequency, heat transfer is peaked.

Studies above shows that the structure of geometry where pulsating flow passes through is a key parameter for the rate of heat transfer. Complex geometries like grooved surfaces provide better heat transfer performances under pulsating flow conditions. The present study is motivated to investigate heat transfer enhancement potential of pulsating flow in a sinusoidal grooved channel when there is $0^{\circ}$ phase shift between sinusoidal walls. In order to achieve this numerical simulations are performed by solving the governing equations using finite volume approach.

\section{PROBLEM DESCRIPTION AND NUMERICAL METHOD}

\subsection{Definition of Problem}

Schematic view of the channel geometry used in the present study is given in Figure 1. The channel is consist of adiabatic flat sections and constant temperature wavy section. Temperature of wavy section $(T)$ is differentially higher than inlet temperature of fluid $\left(\mathrm{T}_{\mathrm{i}}\right)$.

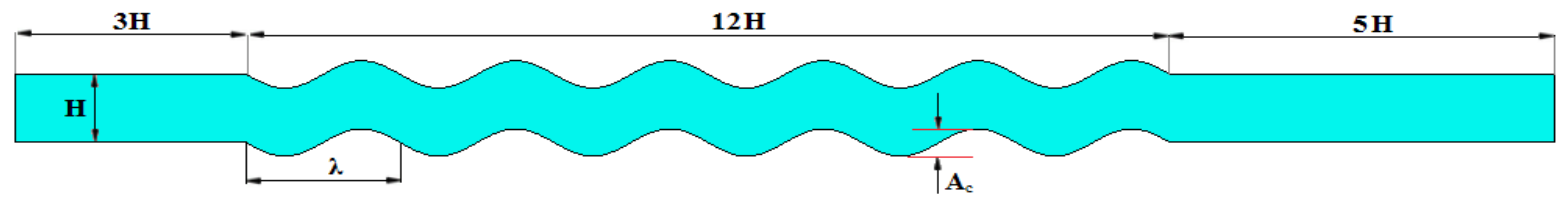

Figure. 1. Schematic view of channel.

Distance between channel walls $(\mathrm{H})$ is considered as $2 \mathrm{~cm}$ and profiles of wavy wall defined by:

$$
y=A_{C}\left[\sin \left(2 \pi \gamma \frac{x-x_{s}}{x_{e}-x_{s}}\right)\right]
$$

Here $\mathrm{x}_{\mathrm{e}}$ and $\mathrm{x}_{\mathrm{s}}$ represent the starting and ending position of wavy section. Amplitude of wavy wall (Ac) kept constant and its value is $0.2 . \gamma$ is undulation number.

\subsection{Governing Equations}

In this study the fluid is taken to be Newtonian, incompressible and its Prandtl number (Pr) is equal to 6.93. The flow is laminar, two dimensional and unsteady. Additionally, gravity effect and thermal radiation are neglected. Under these circumstances non-dimensional form of governing equations becomes,

Continuity equation:

$$
\frac{\partial \mathrm{U}}{\partial \mathrm{x}}+\frac{\partial \mathrm{V}}{\partial Y}=0
$$


Momentum equation:

$$
\begin{aligned}
& \frac{\partial \mathrm{U}}{\partial \tau}+\mathrm{U} \frac{\partial \mathrm{U}}{\partial \mathrm{X}}+\mathrm{V} \frac{\partial \mathrm{U}}{\partial \mathrm{Y}}=-\frac{\partial \mathrm{P}}{\partial \mathrm{X}}+\frac{1}{\operatorname{Re}}\left(\frac{\partial^{2} \mathrm{U}}{\partial \mathrm{X}^{2}}+\frac{\partial^{2} \mathrm{U}}{\partial \mathrm{Y}^{2}}\right) \\
& \frac{\partial \mathrm{V}}{\partial \tau}+\mathrm{U} \frac{\partial \mathrm{V}}{\partial \mathrm{X}}+\mathrm{V} \frac{\partial \mathrm{V}}{\partial \mathrm{Y}}=-\frac{\partial \mathrm{P}}{\partial \mathrm{Y}}+\frac{1}{\operatorname{Re}}\left(\frac{\partial^{2} \mathrm{~V}}{\partial \mathrm{X}^{2}}+\frac{\partial^{2} \mathrm{~V}}{\partial \mathrm{Y}^{2}}\right)
\end{aligned}
$$

Energy equation:

$$
\frac{\partial \theta}{\partial \tau}+U \frac{\partial \theta}{\partial X}+V \frac{\partial \theta}{\partial Y}=\frac{1}{\operatorname{RePr}}\left(\frac{\partial^{2} \theta}{\partial X^{2}}+\frac{\partial^{2} \theta}{\partial Y^{2}}\right)
$$

In these equations dimensionless parameters are:

$$
\begin{aligned}
& (U, V)=\frac{(u, v)}{u_{0}}, \quad(X, Y)=\frac{(x, y)}{H}, P=\frac{p}{\rho u_{o}^{2}}, \quad \theta=\frac{T-T_{i}}{T_{s}-T_{i}}, \quad \tau=\frac{t H}{u_{o}}, \quad R e=\frac{\rho u H}{\mu} \\
& \operatorname{Pr}=\frac{C_{p} \mu}{k} \\
& \text { Local: } \mathrm{Nu}=\left(\frac{-H}{T_{s}-T_{i}}\right)\left(\left.(\partial T / \partial n)\right|_{\text {BottomWall }}+\left.(\partial T / \partial n)\right|_{\text {Top Wall }}\right) \\
& \text { Space averaged: } \mathrm{Nu}_{\bar{x}}=\frac{1}{\left(x_{\varepsilon}-x_{s}\right)} \int_{x_{s}}^{x_{\varepsilon}} N u d s \\
& \text { Space and period averaged: } N u_{\bar{\tau}}=\frac{1}{\tau_{p}} \int_{0}^{2 \pi} N u_{\bar{x}} d \tau
\end{aligned}
$$

Local, space-averaged, space and period timed-averaged Nusselt numbers $(\mathrm{Nu})$ are used for estimation of convection rate from wavy wall. For calculation of the Nusselt number $(\mathrm{Nu})$ instead of bulk temperature, inlet temperature is chosen as a reference parameter [14].

\subsection{Boundary Conditions}

Fully developed Poiseuille flow with added sinusoidal pulsation term is applied at the channel inlet. Inflow temperature is uniform and steady. Non-dimensional form of inlet velocity profile is presented as:

$$
\mathrm{U}(\mathrm{Y}, \tau)=\frac{3}{2} \mathrm{u}_{0}\left(1-(\mathrm{Y}-1)^{2}\right)\left[1+\mathrm{A}_{\mathrm{p}} \sin (2 \pi \mathrm{St} \tau)\right], \mathrm{V}=0, \theta=0
$$

In equation (9) $A_{p}$ is non-dimensional amplitude, St is the Strouhal number which represents non-dimensional frequency and it is defined as $\mathrm{St}=\mathrm{fH} /$ uo. The no-slip boundary condition available for channel walls $\mathrm{U}=\mathrm{V}=0$. Straight sections of walls are adiabatic, wavy walls has constant temperature. At the exit of the channel, temperature and velocity gradients are equal to zero , , .

\subsection{Numerical Procedure and Code Validation}

Finite volume method is employed for discretization of governing equations. Discretization is second order accurate and SIMPLE algorithm used for velocity-pressure coupling. Time step size is set as 0.001 second. For grid

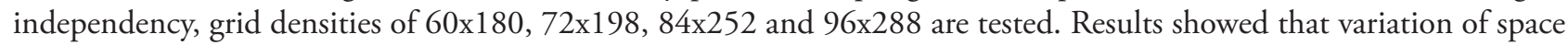
averaged Nusselt number () is around 3\% between $84 \times 252$ and 96x288. Therefore 84x252 grid used in wavy channel. The convergence criterion for continuity, momentum and energy equations is determined as $10^{-5}$. The numerical procedure is validated by applying it for the problem defined in the study of Wang and Chen [15]. Agreement between results is presented in Fig. 2. 


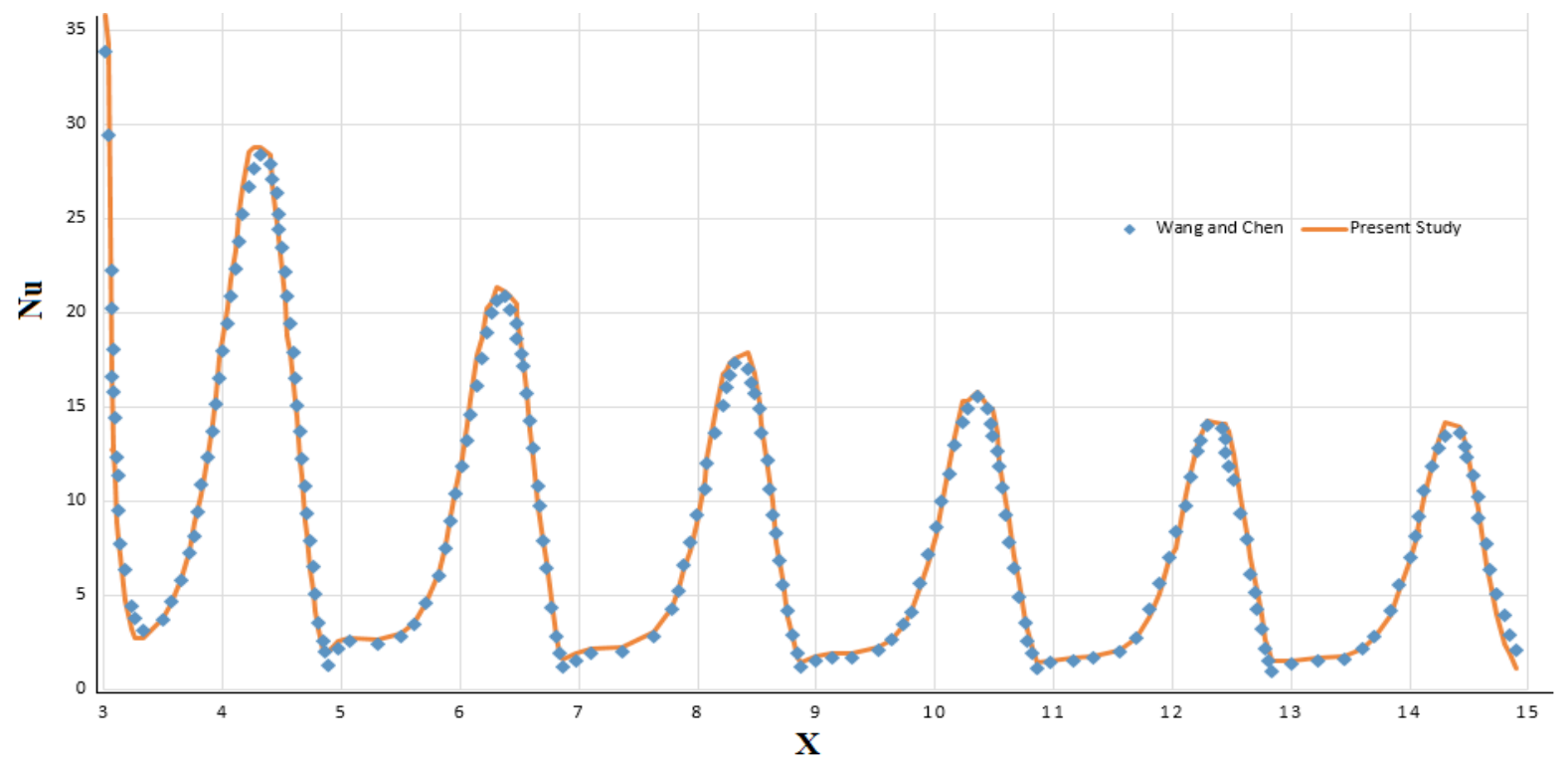

Figure 2. Distribution of Nusselt number $(\mathrm{Nu})$ along bottom wavy wall for $\mathrm{Re}=500$ and $\mathrm{A}_{\mathrm{c}}=0.2$.

\section{RESULTS AND DISCUSSIONS}

Figure 4 shows velocity vectors for steady flow and four different phase angle of pulsating flows. Dimensionless form of inlet mean velocity that corresponds these phase angles can be observed in Figure 5. From the velocity vectors it can be seen that there are vortices trapped inside of grooves. For steady flow, interaction between these vortices and main stream is poor, in other words, mixing between fluid inside grooves and fluid in core region is not effective. In this case high temperature fluid trapped inside grooves.

As it is shown in Figures 4b,c,d and e, flow structures in pulsating flow are quite different from a steady case. At the beginning of the pulsation period $(\omega \mathrm{t}=0)$, the flow inside grooves is not dominated by a vorticity concentration. There is fluid transfer between the region of grooves and main stream. Afterwards, a complete vortices are developed and rotational speeds raise depending on the flow pulsation period shown in Figures $4 \mathrm{c}$ and d. Finally, vorticity concentrations reach their maximum size when phase angle becomes $3 \Pi / 2$, at this state velocity magnitude of flow inside groove is higher as compared to steady flow. As it can be seen in Figure 4e these vortices intensely disturb the main stream. Then they dissipate and flow returns their initial state given in Figure 4a. This situation successively repeats and provides better fluid mixing also prevents trapping of hot fluid inside of grooves.

Temperature distribution in wavy channel for pulsating and steady flow is given in Figure 6. From the figure it can be clearly seen that there is a considerable temperature differences between grooves and area close to central axis for the steady flow. But in pulsating flow hot fluid inside grooves diffuses to the cold region. Consequently, the temperature gradient between channel walls and fluid is increased and heat transfer is enhanced.

Variation of inlet dimensionless velocity $(\mathrm{U})$ with respect to the time causes that the space averaged Nusselt number () varies periodically. For different Strouhal numbers (St), the variation of space averaged Nusselt number for wavy walls is demonstrated in Figure 7. As it is seen in the figure there is a strong relationship between pulsation dimensionless velocity (U) profile and the Nusselt number (). Just like a pulsation of inlet flow, the Nusselt number () reduces at deceleration phase and it increases at acceleration phase. It is obviously seen in figure 7 when Strouhal numbers (St) are equal to 0.15 and 0.25 even the smallest value of the Nusselt number () is higher than the steady case. But for the Strouhal number (St) the value of 0.05 , the Nusselt number () becomes lower than the steady case when the inlet velocity decreases; even so, the space and time-averaged Nusselt number () is still higher than the steady case. 


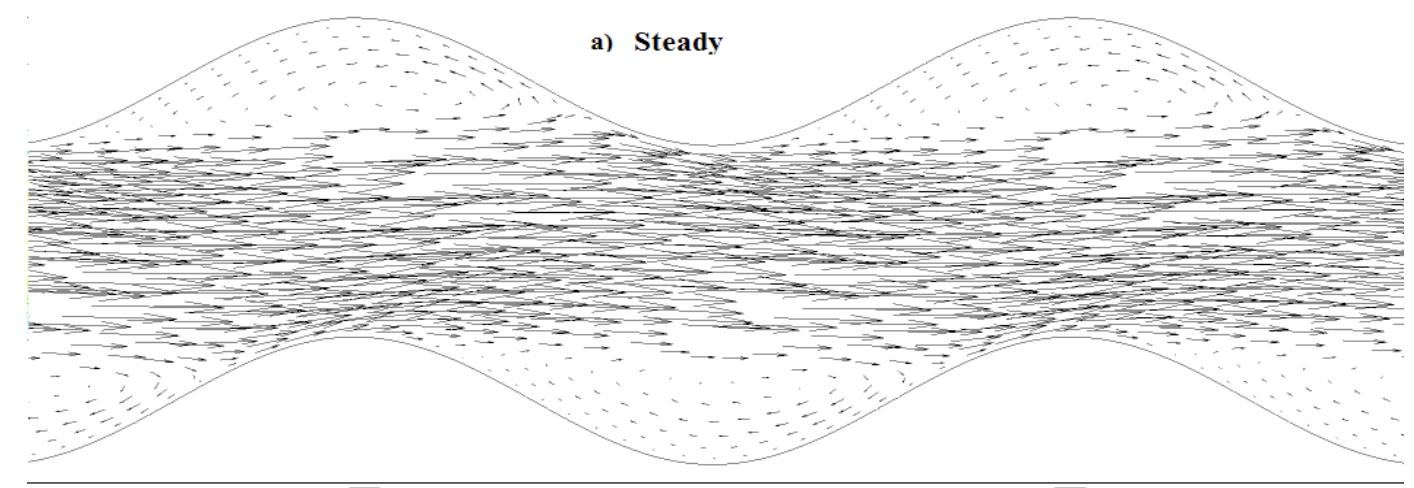

b) 2 nп+0
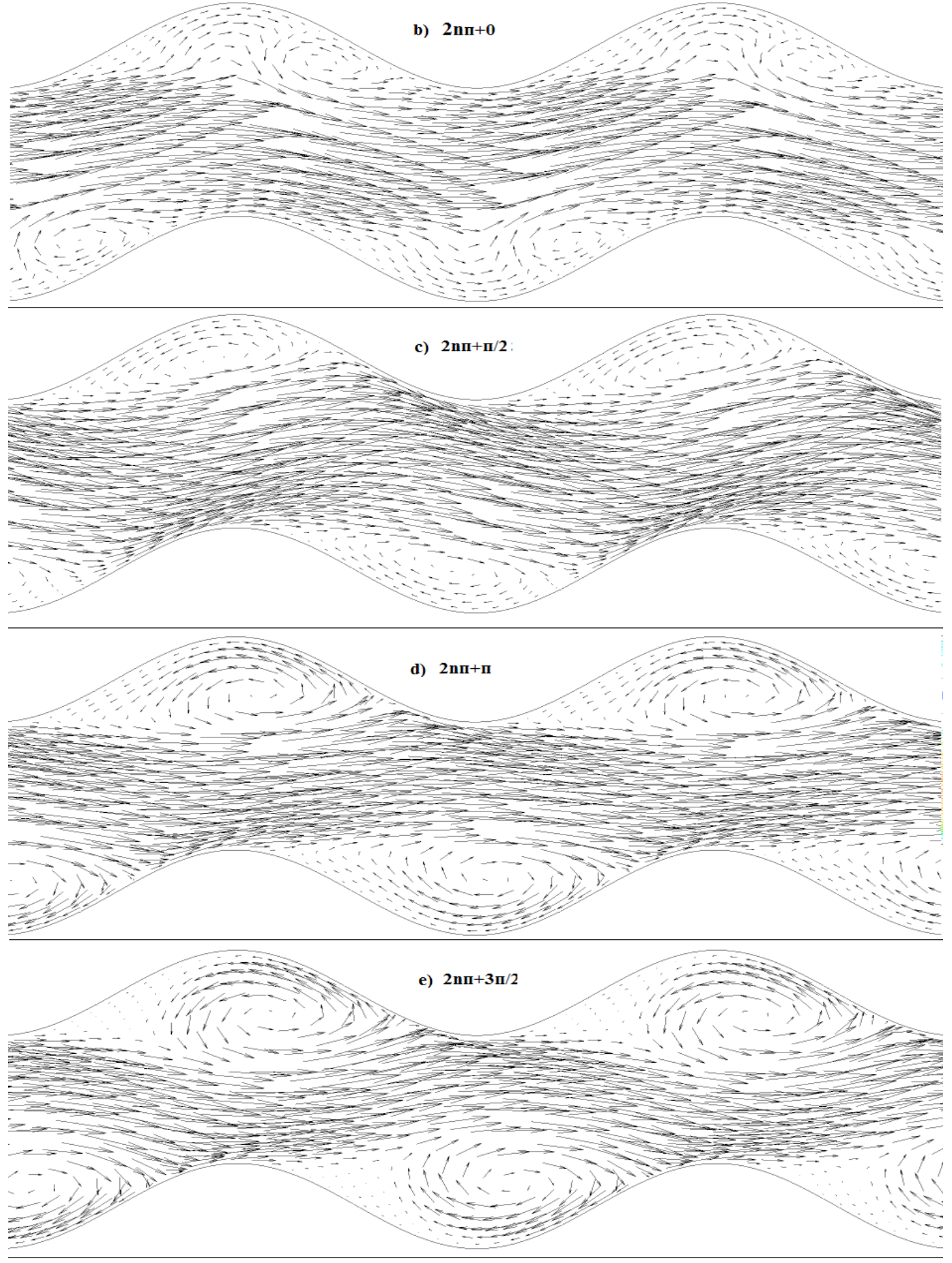

Figure 4. Velocity vectors (V) for steady and pulsating flow at $R e=400$ and $S t=0.25$. 


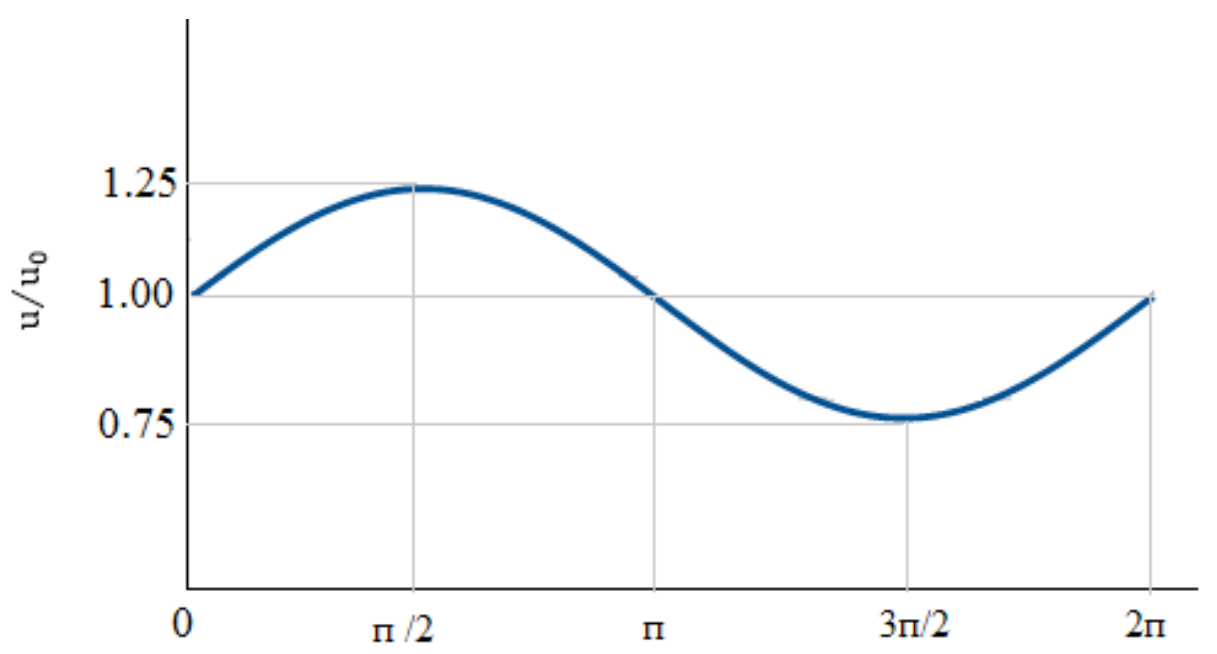

Figure 5 Variation of dimensionless inlet velocity $\left(\mathrm{u} / \mathrm{u}_{0}\right)$ over a period of cycle

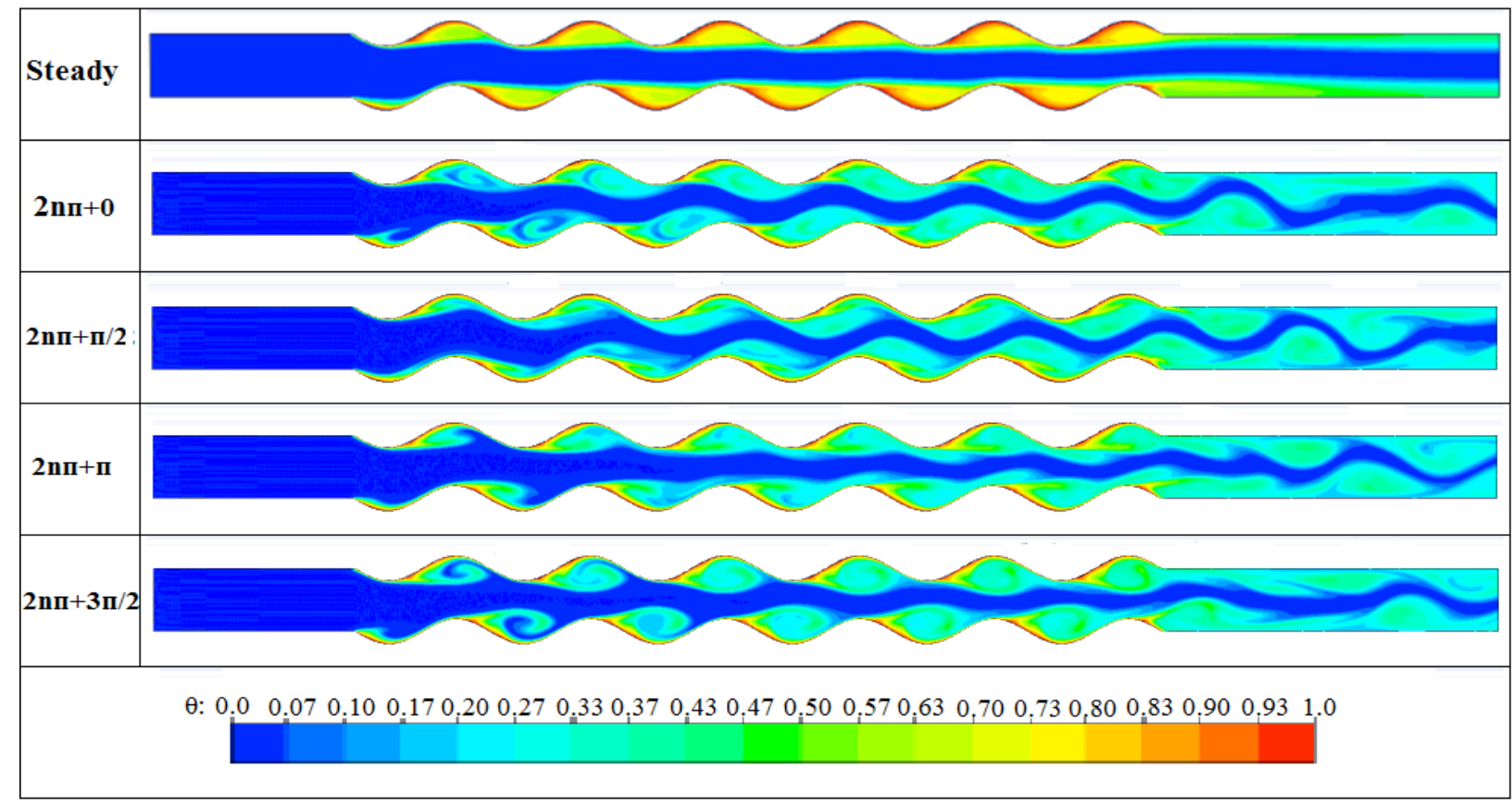

Figure 6 Temperature distribution inside channel for steady and pulsating flow at $\operatorname{Re}=400, \mathrm{St}=0.25$

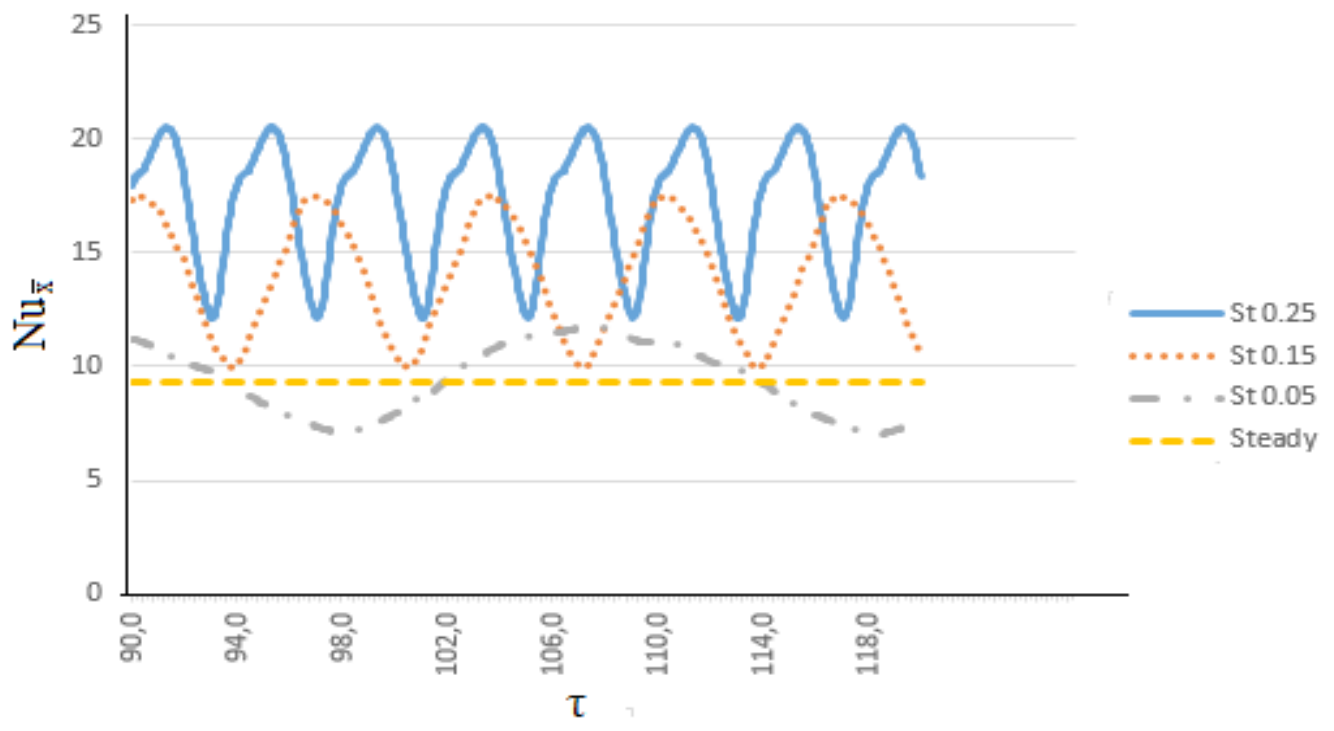

Figure. 7. Variation of the Nusselt number () with time at different Strouhal numbers (St) and $\quad \operatorname{Re}=400$ 
In order to investigate heat transfer performance of pulsating flow, the time averaged Nusselt number values are obtained and presented in Figures 8 and 9. Time-averaged Nusselt numbers are calculated when the flow regime reaches the periodic state. Heat transfer enhancement ratio of pulsating flow $(\mathrm{St}>0)$ can be seen from Figure 8 by comparing the Nusellt number () value with the steady case $(\mathrm{St}=0)$.

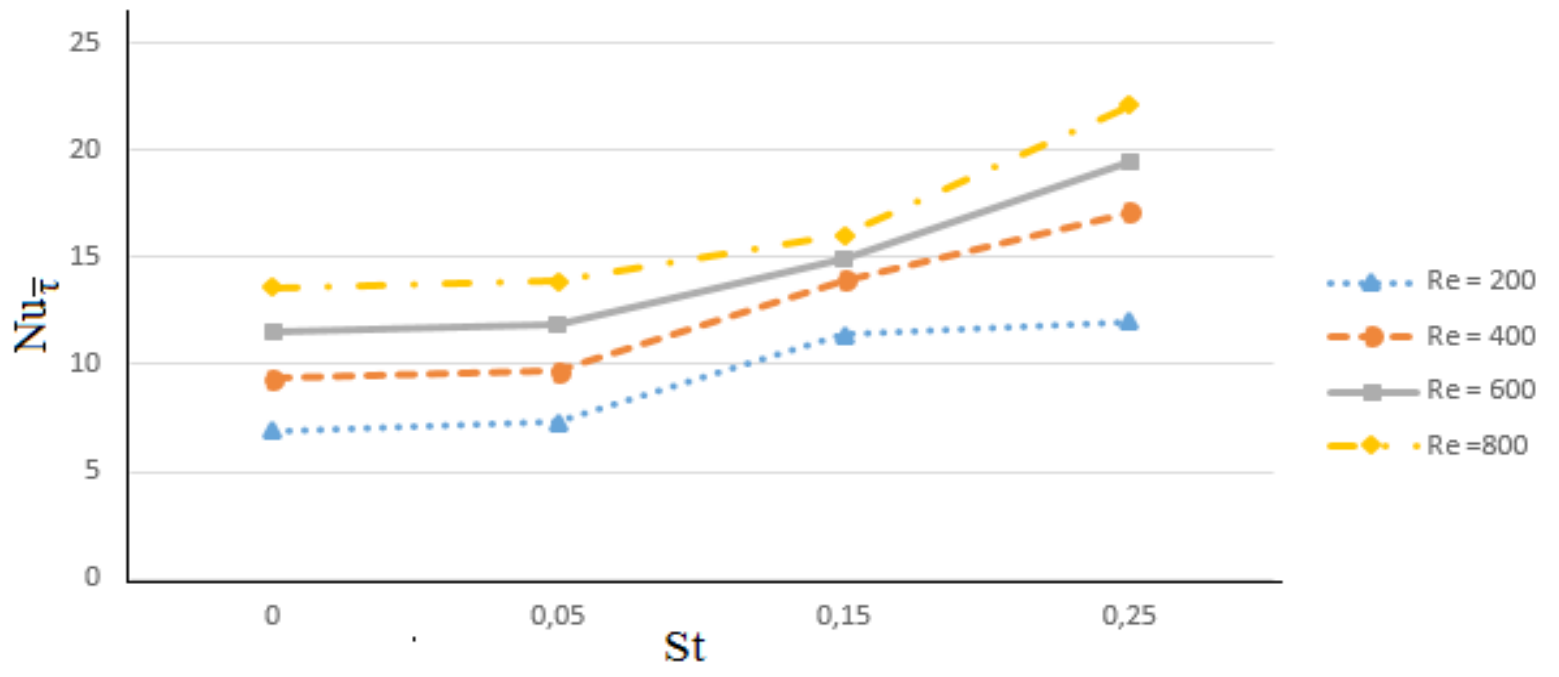

Figure. 8 Time-averaged Nusselt number $(\mathrm{Nu})$ values at various strouhal numbers $(\mathrm{St})$.

Enhancement of heat transfer is noteworthy for the Strouhal number (St) value of 0.05. At this value of the Strouhal number (St), an increase of heat transfer rate is around 6\% and the Reynolds number does not cause big difference. This situation can also be observed from Figure 9. There are not a wide range differences between Nusselt numbers () based on strouhal numbers, $\mathrm{St}=0$ and $\mathrm{St}=0.05$. The effect of pulsating flow on heat transfer becomes more evident when the Strouhal number $(\mathrm{St})$ is equal to 0.15 . Heat transfer enhancement rate which goes upto $65 \%$ as comparing to the steady flow case and after that the effect of Reynolds number becomes important. As it is seen in Figure 9 enhancement heat transfer ratio is reduced when the Reynolds number is lower than 400 . With the further increase of Strouhal number (St) the heat transfer enhancement ratio continues to increase. When the Strouhal number ( $\mathrm{St}$ ) is equal to 0.25 the Nusselt number () value increases up to $82 \%$.

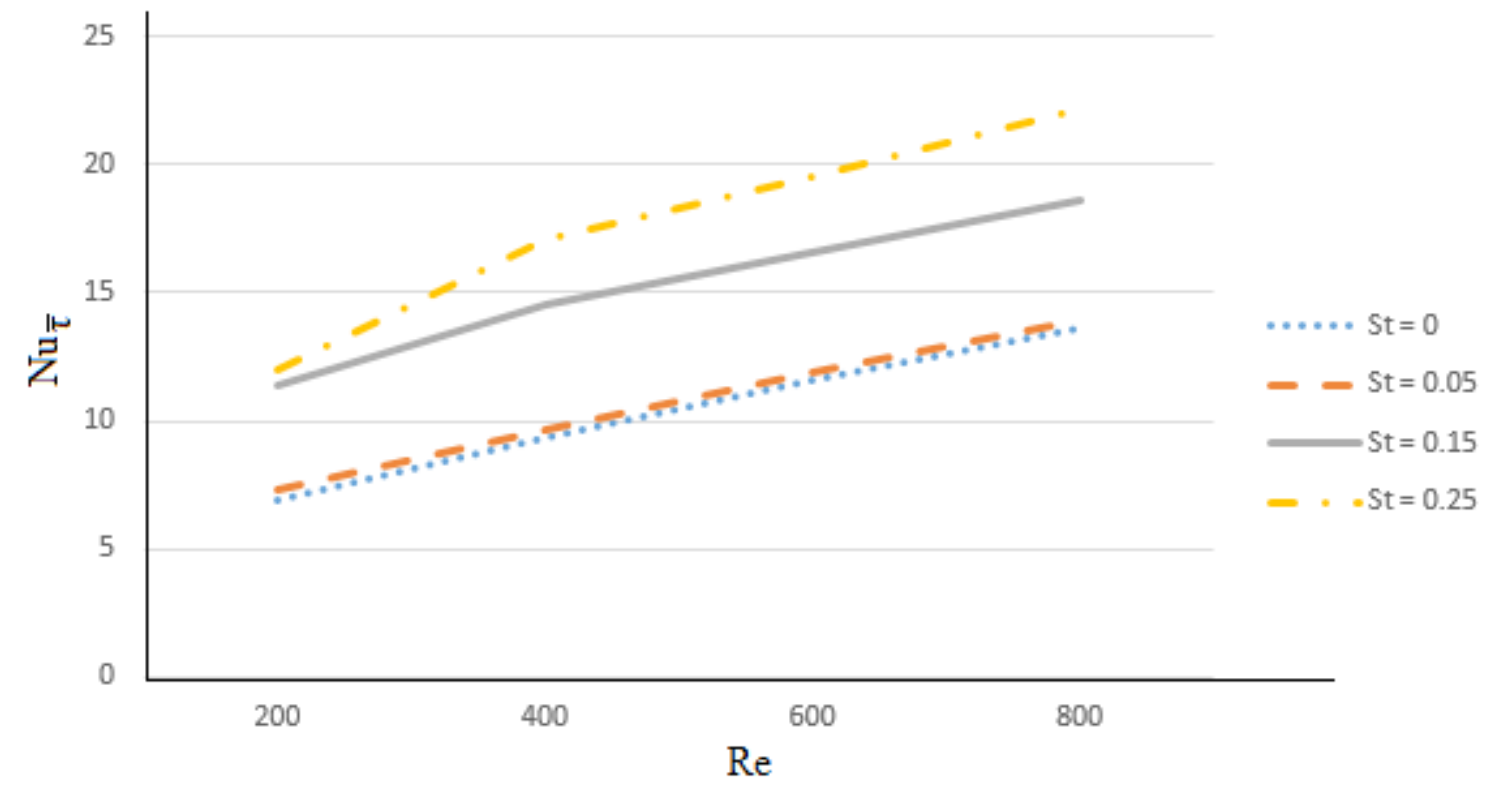

Figure 9. Time-averaged Nusselt number () values at various Reynolds numbers.

\section{CONCLUSION}

Pulsating flow and heat transfer through wavy channel numerically simulated for and . Variation of inlet dimensionless velocity (U) with respect to time causes that the Nusselt number () varies periodically. Time-averaged Nusselt numbers 
(St) show that pulsating flow has positive contribution on the heat transfer rate for the geometry used in this study. The rate of heat transfer is strongly affected by the Strouhal number (St). Heat transfer enhancement is not significant and the effect of the Reynolds number is limited for the Strouhal number of $\mathrm{St}=0.05$. This situation changes with increasing the Strouhal number $(\mathrm{St})$; heat transfer enhancement gets better and the role of the Reynolds number becomes more dominant.

\section{ACKNOWLEDGEMENT}

The authors would like to express their appreciation to Ç.Ü. Scientific Research Project Department for their financal support (Project ID; FDK-2016-6952).

\section{REFERENCES}

[1] Guo, Z. and Sung, H.J. (1997). Analysis of the Nusselt number in pulsating pipe flow. International journal of heat and mass transfer, vol. 40, no. 10, pp. 2486-2489. 10.1016/S0017-9310(96)00317-1

[2] Chattopadhyay, H., Durst, F., and Ray, S. (2006). Analysis of heat transfer in simultaneously developing pulsating laminar flow in a pipe with constant wall temperature. International communications in heat and mass transfer, vol. 33, no. 4, pp. 475-481. 10.1016/j.icheatmasstransfer.2005.12.008

[3] Rahgoshay, M., Ranjbar, A.A., and Ramiar, A. (2012). Laminar pulsating flow of nanofluids in a circular tube with isothermal wall. International Communications in Heat and Mass Transfer, vol. 39, no. 3, pp. 463-469. 10.1016/j.icheatmasstransfer.2011.12.008

[4] Wang, X. and Zhang, N. (2005). Numerical analysis of heat transfer in pulsating turbulent flow in a pipe. International Journal of Heat and Mass Transfer, vol. 48, no. 19, pp. 3957-3970. 10.1016/j.ijheatmasstransfer.2005.04.011

[5] Mehta, B. and Khandekar, S. (2015). Local experimental heat transfer of single-phase pulsating laminar flow in a square mini-channel. International Journal of Thermal Sciences, vol. 91, pp. 157-166. 10.1016/j.ijthermalsci.2015.01.008

[6] Moon, J.W., Kim, S.Y., and Cho, H.H. (2005). Frequency-dependent heat transfer enhancement from rectangular heated block array in a pulsating channel flow. International journal of heat and mass transfer, vol. 48, no. 23, pp. 4904-4913. 10.1016/j.ijheatmasstransfer.2005.06.006

[7] Ji, T.H., Kim, S.Y., and Hyun, J.M. (2008). Experiments on heat transfer enhancement from a heated square cylinder in a pulsating channel flow. International Journal of Heat and Mass Transfer, vol. 51, no. 5, pp. 1130-1138. 10.1016/j.ijheatmasstransfer.2007.04.015

[8] Kim, S.Y., Kang, B.H., and Jaluria, Y. (1998). Thermal interaction between isolated heated electronic components in pulsating channel flow. Numerical Heat Transfer, Part A Applications, vol. 34, no. 1, pp. 1-21. Doi 10.1080/10407789808913974

[9] Jin, D., Lee, Y., and Lee, D.-Y. (2007). Effects of the pulsating flow agitation on the heat transfer in a triangular grooved channel. International journal of heat and mass transfer, vol. 50, no. 15, pp. 3062-3071. 10.1016/j.ijheatmasstransfer.2006.12.001

[10] Nandi, T.K. and Chattopadhyay, H. (2013). Numerical investigations of simultaneously developing flow in wavy microchannels under pulsating inlet flow condition. International Communications in Heat and Mass Transfer, vol. 47, pp. 27-31. 10.1016/j.icheatmasstransfer.2013.06.008

[11] Akdag, U., Akcay, S., and Demiral, D. (2014). Heat transfer enhancement with laminar pulsating nanofluid flow in a wavy channel. International Communications in Heat and Mass Transfer, vol. 59, pp. 17-23.

[12] Jafari, M., Farhadi, M., and Sedighi, K. (2014). Heat transfer enhancement in a corrugated channel using oscillating flow and nanoparticles: an LBM approach. Numerical Heat Transfer, Part A: Applications, vol. 65, no. 6, pp. 601-626. $10.1080 / 10407782.2013 .836023$

[13] Alawadhi, E.M. and Bourisli, R.I. (2009). The role of periodic vortex shedding in heat transfer enhancement for transient pulsatile flow inside wavy channels. Int. Journal of Natural Sciences and Engineering, vol. 1, no. 2, pp. 79-85.

[14] Chattopadhyay, H., Durst, F., and Ray, S. (2006). Analysis of heat transfer in simultaneously developing pulsating laminar flow in a pipe with constant wall temperature. International communications in heat and mass transfer, vol. 33, no. 4, pp. 475-481. 10.1016/j.icheatmasstransfer.2005.12.008

[15] Wang, C.-C. and Chen, C.-K. (2002). Forced convection in a wavy-wall channel. International Journal of Heat and Mass Transfer, vol. 45, no. 12, pp. 2587-2595. 